\title{
Compressively Sensed Video Acquisition in Transmission Electron Microscopy
}

\author{
Bryan W. Reed ${ }^{1}$, Sang Tae Park ${ }^{1}$, Ruth S. Bloom ${ }^{1}$, and Daniel J. Masiel ${ }^{1}$ \\ 1. Integrated Dynamic Electron Solutions, Inc., Pleasanton, CA 94588, USA
}

Transmission electron microscopy (TEM) is an extremely powerful tool for physical, material, and biological science at the nanoscale. This is especially true for higher-dimensional acquisition modes that produce data beyond the usual two-dimensional images, adding resolution in time, depth (via tomography), scattering angle (through STEM-diffraction, also called 4D STEM), and spectroscopy. Unfortunately, these higher-dimensional modes are extremely bandwidth-hungry. Every added dimension greatly multiplies the number of bytes that need to be captured, with associated increases in acquisition time, sample damage, and equipment expense. This includes, more and more, the equipment needed to transfer, store, and analyze the data. Modern kilohertz-scale cameras are starting to address the issue, but they are not a complete solution, and they can be quite expensive.

Yet much of the raw-byte-count data so acquired is either redundant or practically meaningless. Standard analysis methods such as principal component analysis can often eliminate $90 \%$ or more of these bytes without losing any information of interest to the user. Even then, the data is usually highly compressible, with strong multi-length-scale spatial correlations allowing standard image compression schemes to reduce the byte counts still further.

Compressive sensing (CS) addresses this problem by posing the question: Why acquire the redundant information in the first place? CS, by performing the data acquisition in a nonstandard way, effectively performs data compression in the analog domain, before the analog-to-digital conversion bottleneck. This speeds up acquisition, reduces data transfer and storage costs, and potentially allows a given piece of hardware to achieve resolution (time, spectral, etc.) well beyond what it would normally be capable of. Decompression is accomplished through any of a variety of algorithms, ranging from $l_{1}$-norm regularization in a known sparsity-inducing representation, to total-variation (TV) minimization, to Bayesian overcomplete dictionary learning $[1,2]$.

We have developed a new approach for CS video acquisition in a TEM that uses the same kind of postsample deflection used in movie mode dynamic TEM [3]. A fast, arbitrarily programmable electrostatic deflector placed after the projector lens rasters the TEM image or diffraction pattern into an array of images (usually $3 \times 3$ or $4 \times 4$ ) on a large camera (Figure 1). This allows multiple images to be captured in a single camera acquisition, even in a sequential non-CS operating mode. When the images are exposed in a known pseudorandom sequence, returning to each image multiple times in one camera exposure, this in effect performs data compression on the video sequence. CS techniques can then reconstruct $\sim 50$ or more frames of kilohertz-scale video in a single acquisition from a conventional slow-scan camera, as we have demonstrated on three different test systems (Figure 2 shows a test case taken on the system we installed at Sandia National Laboratory in collaboration with K. Hattar et al.[5]). Coupled with modern high-frame-rate cameras, the time resolution could be even faster, ultimately limited only by beam current and signal-to-noise ratio limitations and not by the data collection system itself.

This kind of rapid acquisition of images has clear applications in in situ TEM experimentation, tomography, STEM-diffraction, and potentially spectrum imaging. Moreover, the system is simple and 
modular and the installation is quick and non-invasive, requiring no modification of the TEM itself. Integrating the deflection system with the TEM camera system has proven surprisingly easy, such that once the system is configured and aligned, a time-compressed measurement is performed simply by acquiring an image in the standard way. The deflection system can also be easily retracted, returning the TEM to its standard operating mode in a matter of seconds.

\section{References:}

[1] E. J. Candes and T. Tao, IEEE Trans. Information Theory 52 (2006), p. 5406.

[2] M. Zhou et al, IEEE Trans. Image Proc. 21 (2012), p. 130.

[3] T. LaGrange, B. W. Reed, and D. J. Masiel, MRS Bull. 40 (2015), p. 22.

[4] This material is based upon work supported by the U.S. Department of Energy, Office of Science, Office of Basic Energy Sciences, under Award Number DE-SC0013104.

[5] Sandia National Laboratories is a multi-program laboratory managed and operated by Sandia Corporation, a wholly owned subsidiary of Lockheed Martin Corporation, for the U.S. Department of Energy's National Nuclear Security Administration under contract DE-AC04-94AL85000.

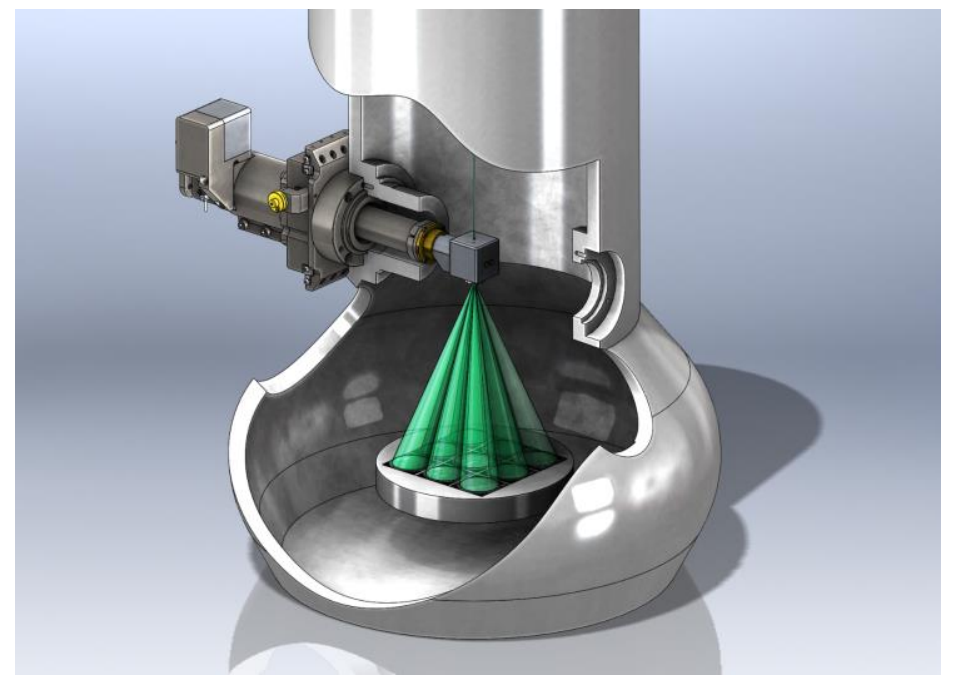

Figure 1. The deflector is inserted into a standard detector port. A fast switching circuit controlled by an arbitrarily programmable digital pattern generator sends images to multiple subregions on a camera, rapidly jumping back and forth from one image position to another, in a way that encodes up to 50 frames of video in one exposure.

Figure 2. An example of reconstructed time-compressed data, producing 50 video frames of a uniformly moving grid from one camera exposure.

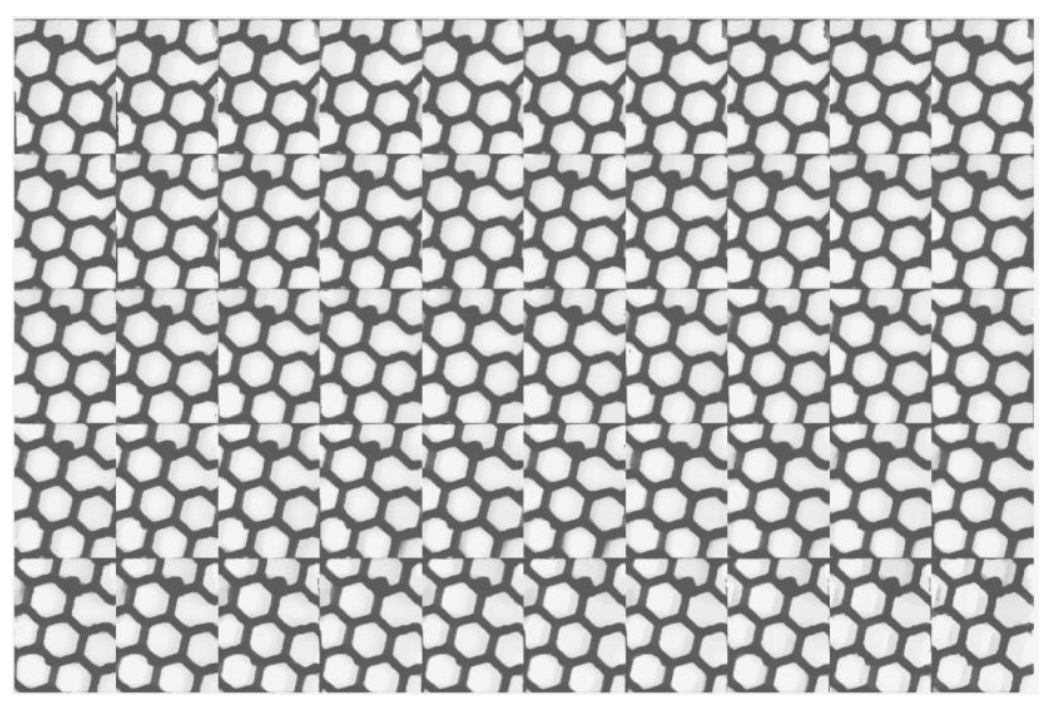

